\title{
Investigation of the Toxicity of Amine-coated, Carboxyl-coated and Polyaniline-coated FeO Magnetic Nanoparticles in Caenorhabditis elegans
}

Callaway MK' ${ }^{1}$, Ochoa JM${ }^{1}$, Perez EE 1 , Ulrich PE ${ }^{1}$, Alocilja EC ${ }^{2}$ and Vetrone SA ${ }^{1 *}$

${ }^{1}$ Department of Biology, Whittier College, 13406 E. Philadelphia St., Whittier, CA 90608, USA

${ }^{2}$ Department of Biosystems and Agricultural Engineering, Michigan State University, USA

\begin{abstract}
Since the introduction of nanotechnology there has been an increase in the use of nanoparticles (NPs) in the development of biosensors for the detection of bacterial pathogens. Consequently, research exploring their potential toxicity has also increased as some have been shown to be harmful depending on their size, shape, and chemical composition making it imperative that we understand their possible harm to humans and the environment. In this study we investigated the potential toxicity of three differently coated FeO Magnetic NPs (MNPs), amine, carboxyl, and polyaniline, on the Caenorhabditis elegans (C. elegans) nematode. Briefly, C. elegans were exposed to the singular coated-MNPs types at a concentration of $100 \mu \mathrm{g} / \mathrm{mL}$ and assessed for physiological effects on their metabolism, reproduction, longevity, and oxidative stress resistance. Exposure to singular coated-MNPs corresponded with a statistical decrease in their metabolic and acute oxidative stress resistance abilities, and revealed a trend towards lower reproduction and longevity. Taken together, these results add to the growing evidence that FeO coated-MNPs have an in vivo toxic effect on $C$. elegans. These findings advocate for a need to take safety precautions when discarding FeO coated-MNPs as they may pose a toxic health hazard to our environment and health.
\end{abstract}

Keywords: Coated-FeO nanoparticles; Nanotoxicity; Toxicity; C. elegans; Oxidative stress; Longevity; Metabolism; Fertility

\section{Introduction}

The introduction of nanotechnology has lead to an increase in the research and industrial application of NPs in fields related to energy, medicine, safety, and defense [1]. In particular, since the 2001 distribution of Bacillus anthracis through the United States postal system, much attention has been directed towards the use of DNA or antibody based biosensors for the biological detection of pathogens that incorporate the use of NPs [2-5]. Consequently, the more these biosensors are synthesized and used, the larger impact they may have on our environment as they start to accumulate as waste and decompose. Hence, there is a growing need to investigate and monitor the potential nanotoxicity of NPs to both our health and environment. Towards that end, some studies have shown that NPs, such as platinum NPs, are beneficial in their ability to reduce oxidative stress [6], however many others have shown NPs to reduce this ability [7-9]. Conversely, some studies have shown that the NPs themselves can impart toxicity, increase cellular oxidative stress, and damage [10]. For instance, many toxicological studies have been conducted using the C. elegans soil nematode as they would most likely be in first contact with decomposing NPs at waste sites. For example, using the C. elegans animal model it has been revealed that NPs synthesized from various materials, such as zinc, aluminum, titanium, silver, gold, and silica to name a few, can cause harmful effects on living organisms such as reduced egg production, number of off spring, and growth [11-14]. In particular, a study comparing the toxicity of silver-NPs to silver ions, found that while silver ion exposure did not impact growth, it resulted in reduced reproduction potential as seen with silver-NP exposure, although the silver-NP exposure had bigger reduction in reproduction [11].

Another important NP that is increasingly used in the development of biosensors is the Magnetic Nanoparticle (MNP) [15]. Many of these MNPs are synthesized from $\mathrm{FeO}$ (i.e., iron III oxide or ferric oxide) and contain a shell coating that serves as a binding surface. Not only have the MNPs been shown to be toxic by both in vivo and in vitro studies [16], but also a recent study has demonstrated that the Dimercaptosuccinic Acid (DMSA) shell coating of a FeO MNP additionally had toxic properties [17]. Therefore, this study aims to investigate the toxic potential of three differently coated $\mathrm{FeO}$-MNPs (amine-MNPs, carboxyl-MNPs, and polyaniline-MNPs) that have been used in the development of DNA and antibody based biosensors for the detection of bacterial pathogens $[2-5,18,19]$. Using the $C$. elegans model, that has been extensively studied in toxicological investigations from the molecular to organismal level $[20,21]$, we will evaluate the potential toxicity of these three differently coated-MNPs by assessing their impact on the worm's metabolism, reproduction ability, longevity, and resistance to oxidative stress. We hypothesize that similar to DMSA-coated $\mathrm{FeO}$ MNPs, C. elegans exposure to amine-MNPs, carboxyl-MNPs, and polyaniline-MNPs will result in overall lowered physiological health outcomes.

\section{Materials and Methods}

\section{Nanoparticles}

For these studies we used three different types of coated $\mathrm{FeO}$-MNPs: amine-coated MNPs, carboxyl-coated MNPs, and polyaniline-coated MNPs. The MNPs were synthesized and provided by Dr. Evangelyn C. Alocilja (Michigan State University, East Lansing, MI, USA), which have been characterized previously $[18,19]$. Briefly, coated iron-core MNPs were determined to be approximately between 50 to $100 \mathrm{~nm}$ in

*Corresponding author: Sylvia A. Vetrone, Whittier College, Department of Biology, 13406 E. Philadelphia St., Whittier, CA 90608, USA, Tel: +1 562904 4200; E-mail: svetrone@whittier.edu

Received September 03, 2013; Accepted November 27, 2013; Published December 04, 2013

Citation: Callaway MK, Ochoa JM, Perez EE, Ulrich PE, Alocilja EC, and Vetrone SA, et al. (2013) Investigation of the Toxicity of Amine-coated, Carboxyl-coated and Polyaniline-coated FeO Magnetic Nanoparticles in Caenorhabditis elegans. J Biosens Bioelectron 4: 145. doi: 10.4172/2155-6210.1000145

Copyright: (c) 2013 Callaway MK, et al. This is an open-access article distributed under the terms of the Creative Commons Attribution License, which permits unrestricted use, distribution, and reproduction in any medium, provided the original author and source are credited. 
size by transmission electron microscopy. The electrical properties of the MNPs were investigated by four-point probe measurements and $\mathrm{I}-\mathrm{V}$ measurements and had a resistivity of $0.385 \Omega \mathrm{cm}$ and showed ohmic behavior. MNPs were prepared for exposure to C. elegans in the following manner: the coated-MNPs were reconstituted in sterile water, and added to the C. elegans food source Escherichia coli OP50 (OP50) at a concentration of $100 \mu \mathrm{g} / \mathrm{mL}(100 \mathrm{mg} / \mathrm{L})$, as a previous study investigating the toxicity of coated $\mathrm{FeO}$-MNPs demonstrated harmful effects using this concentration [17]. The prepared MNP-OP50 mixture was added to experimental $35 \mathrm{~mm}$ plates containing solid Nematode Growth Media (NGM) seeded with a spot $(20 \mu \mathrm{L})$ of MNP-OP50 food mixture (experimental plates).Control $35 \mathrm{~mm}$ plates consisted of NGM seeded with a spot of OP50 only. C. elegans were then placed onto these plates for all studies conducted. Each plate contained 25 worms, and worms were transferred to fresh plates every 3 days.

\section{Maintenance of C. elegans}

Wild type BristolN2 C. elegans were obtained from the Caenorhabditis Genetics Center (University of Minnesota, Minneapolis, MN, USA) and maintained as frozen stock until needed. All synchronized cultures were grown on $60 \mathrm{~mm}$ solid NGM plates seeded with a spot $(100 \mu \mathrm{l})$ of OP50 for food [22]. Reproductive adults were placed onto fresh 60 $\mathrm{mm}$ NGM plates and allowed to lay eggs for 2-4 hrs, producing agesynchronized groups. Working cultures were maintained at $22 \pm 1^{\circ} \mathrm{C}$.

\section{Fertility Assessment}

Egg Production Assessment: Individual 1-day old adults, grown starting from the egg stage on experimental or control plates, were transferred into individual wells on a 96 well microtitier plate containing $20 \%$ bleach (sodium hypochlorite, $\mathrm{NaOCl}$ ) and incubated for $8 \mathrm{~min}$, to dissolve the cuticle. Remaining eggs were counted at 50X magnification using a K400 Motic dissection microscope (Martin Microscope Co., Easley, SC, USA). Each group (experimental and control) contained a total of 24 worms, per trial. A total of 4 trials were conducted. All groups were maintained at $22 \pm 1^{\circ} \mathrm{C}$

Egg Laying Rate Assessment: Individual 1-day adults, grown starting from the egg stage on experimental or control plates were transferred to fresh $35 \mathrm{~mm}$ NGM plates seeded with $20 \mu \mathrm{L}$ of OP50 only, and allowed to lay eggs for $4 \mathrm{hrs}$. At the end of the $4 \mathrm{hr}$ period, eggs released were counted at 50X magnification using a K400 Motic dissection microscope (Martin Microscope Co., Easley, SC, USA). Egg rate was calculated as eggs laid/hr. Each group (experimental and control) contained a total of 4 worms, per trial, and 4 trials were conducted. All groups were maintained at $22 \pm 1^{\circ} \mathrm{C}$.

\section{Defecation Assessment}

Individual 2-day old adults, grown starting from egg stage on experimental or control plates, were transferred to fresh $35 \mathrm{~mm}$ NGM plates seeded with $20 \mu \mathrm{L}$ of OP50 only, and allowed to acclimate for 5 min. Observation of pBoc movements, the interval between the obvious posterior contractions that load the rectum for defecation [23], were recorded over a period of 10 minutes (600s) using a K400 Motic dissection microscope (Martin Microscope Co., Easley, SC, USA). Defecation rate was calculated as the average pBoc movements/600s. Each group contained a total of 4 worms, per trial, and a total of 4 trials were conducted.

\section{Longevity Assessment}

Synchronized worms at 3-days of age (L4 stage) were transferred to $35 \mathrm{~mm}$ experimental or control plates and then transferred daily to fresh plates until the cessation of egg laying to avoid confounding generations. All groups were maintained at $22 \pm 1^{\circ} \mathrm{C}$. Worms were monitored over a 20-day period and scored as dead if they did not respond to a touch stimulus. Animals that showed bagging, exploded or crawled off the plates were censored. Each group contained a total of 100 worms over 4 plates ( 25 worms per plate), per trial. A total of 4 trials were conducted.

\section{Oxidative Stress Resistance Assessment}

Temporary Oxidative Stress Resistance: Synchronized worms at 3-days of age (L4 stage) were transferred to $35 \mathrm{~mm}$ experimental or control plates for $48 \mathrm{hrs}$, after which they were exposed to a temporary oxidative stress environment by being transferred to fresh $35 \mathrm{~mm}$ NGM plates containing a final concentration of $40 \mu \mathrm{M}$ juglone (5-hydroxyl-1, 4-naphthoquinone, Sigma-Adrich) within the $20 \mu \mathrm{L}$ of OP50 and incubated for $24 \mathrm{hrs}$. Juglone is a quinone that generates superoxide anion $\left(\mathrm{O}_{2}^{-}\right)$from molecular oxygen during metabolism [24]. The juglone was added to OP50, mixed, applied directly to the NGM plates which were then allowed to dry for $20 \mathrm{mins}$, after which worms were transferred onto these plates for immediate exposure. Following the exposure, groups were transferred back to fresh corresponding experimental or control plates. Groups were transferred daily until the cessation of egg-laying to avoid confounding generations. Worms were monitored for the next 16 days and scored as dead if they did not respond to a touch stimulus. Animals that showed bagging, exploded or crawled off the plates were censored. All groups were maintained at $22 \pm 1^{\circ} \mathrm{C}$. Each group contained a total of 100 worms over 4 plates $(25$ worms/plate), per trial. A total of 3 trials were conducted.

Acute Oxidative Stress Resistance: Synchronized worms at 3-days of age (L4 stage) were transferred to $35 \mathrm{~mm}$ experimental or control plates for $48 \mathrm{hrs}$ after which they were exposed to an acute oxidative stress environment by transferring worms to $35 \mathrm{~mm}$ plates containing $472 \mu \mathrm{M}$ juglone within the NGM. The plates were prepared by dissolving juglone in $100 \%$ ethanol and immediately mixing it into liquefied NGM at $54^{\circ} \mathrm{C}$. The mixture was poured into plates and after solidification they were seeded with $20 \mu \mathrm{L}$ of OP50 and allowed to dry in a fume hood. Plates were used within $3 \mathrm{hrs}$ of preparation. Survival was monitored every $30 \mathrm{~min}$ for the next $4 \mathrm{hrs}$ after the initial transfer and scored as dead if they did not respond to a touch stimulus. Animals that showed bagging, exploded or crawled off the plates were censored. Each group contained at total of 100 worms over 4 plates ( 25 worms/ plate), per trial. A total of 3 trials were conducted.

\section{Statistical Analysis}

Two-tailed Student's $t$-Test of equal variance was used for all experiments. A p-value $\leq 0.05$ was considered to be statistically significant.

\section{Results}

\section{Impact on Metabolic Health}

As a first step towards determining the potential physiological impact of differently shell coated-MNP exposure on C. elegans, we assessed their ability to defecate normally as previous studies have shown that the defecation rate is a good outcome measure for metabolic health [25-27]. The defecation rate was measured by counting the number of pBoc movements, which represent the second stage of the defecation motor program, over 10 minutes (600s). Our results revealed that while there was no difference in the defecation rate between the control and amine-MNPs groups, there was a statistical decrease in defecation within the carboxyl-MNPs and polyaniline-MNPs groups (Figure 1A) 
Citation: Callaway MK, Ochoa JM, Perez EE, Ulrich PE, Alocilja EC, and Vetrone SA, et al. (2013) Investigation of the Toxicity of Amine-coated, Carboxyl-coated and Polyaniline-coated FeO Magnetic Nanoparticles in Caenorhabditis elegans. J Biosens Bioelectron 4: 145. doi: $10.4172 / 2155-6210.1000145$

Page 3 of 5

A
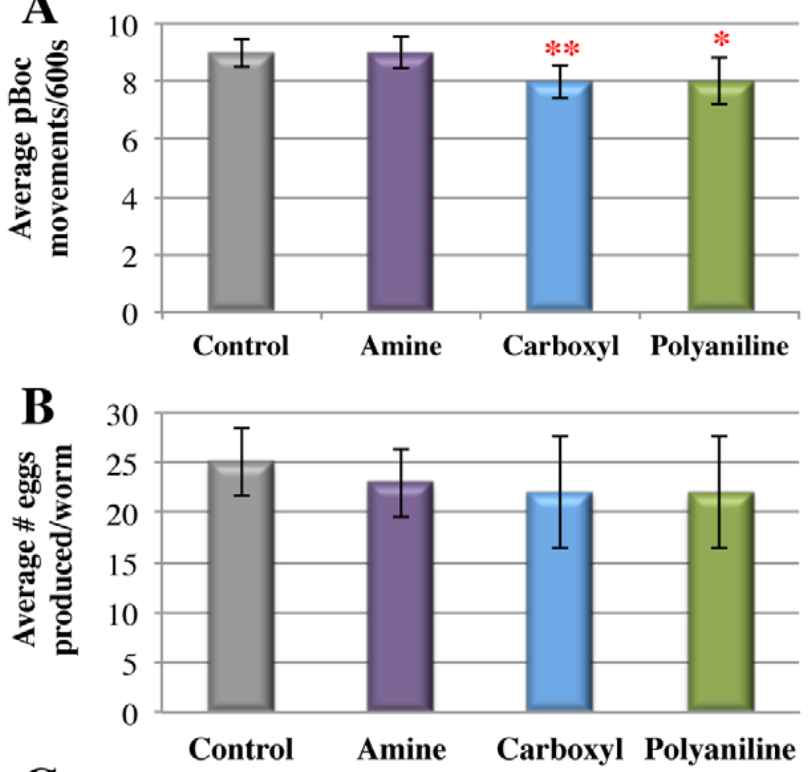

C

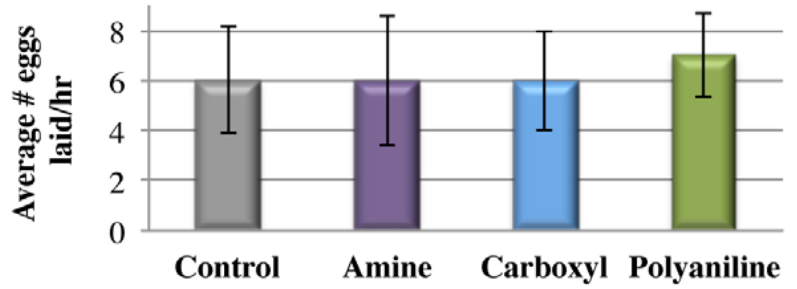

Figure 1: C. elegans fertility and metabolism are not effected by coated MNPs exposure. (A) Defecation rate, pBoc movements per 600s interval. (B) Egg production per nematode. (C) Eggs laid/worm. Each graph is the representative average of 4 different trials. Error bars represent the standard deviation of the average.

${ }^{*}$ represents $p \leq 0.05 ;{ }^{* *}$ represent $p \leq 0.01$, compared with control.

Both the carboxyl-MNPs ( $\mathrm{p}=0.003)$ and polyaniline-MNPs $(\mathrm{p}=0.04)$ exposed groups had a defecation rate of $8 \mathrm{pBoc}$ movements $/ 600 \mathrm{~s}$, which was $11 \%$ below that of the control and amine-MNPs groups, 9 pBoc movements/600s.

\section{Impact on Reproductive Health}

As previous mentioned, MNPs on their own have been shown to have toxic effects [16], and recent studies have also demonstrated their nanoparticles can negatively affect the reproductive abilities of various animal models $[14,28,29]$. Therefore, in order to determine if these differently coated-MNPs had any impact on reproductive health, we conducted two fertility assays on 1-day old adults: eggs produced within a single worms (Figure 1B) and the egg-laying rate (Figure 1C). Exposure of coated-MNPs at $100 \mu \mathrm{g} / \mathrm{mL}$ to C. elegans was not associated with any impact on their ability to produce or lay eggs as compared to controls. MNPs-exposed worms on average contained 22 eggs compared to 25 eggs in controls. Similarly, the average egg-laying rate was $6 \mathrm{eggs} / \mathrm{hr}$ for the control, amine-MNPs, and carboxyl-MPNs groups, while the polyaniline-MNPs had a rate slightly higher, but not significant, of 7 eggs/hr.

\section{Impact on Longevity}

As the exposure to carboxyl-MNPs and polyaniline-MNPs demonstrated an effect on the defecation rate, our next step was to investigate if the coated-MNPs would have any impact on the longevity of the C. elegans, which normally live to approximately 20 days [30]. Beginning at the L4-stage and continuing all they way through the 20day assay, our findings indicate that a continual exposure to coatedMNPs at $100 \mu \mathrm{g} / \mathrm{mL}$ did not correlate with any significant change on longevity (Figure $2 \mathrm{~A}$ ). On average the survival rate at the end of the assay was $18 \%$ for control, $11 \%$ for amine-MNPs, $15 \%$ for carboxylMNPs, and $14 \%$ for polyaniline-MNPs (Figure 2B).

\section{Impact on Oxidative Stress Resistance}

Since oxidative stress is a strong contributor to reduced health, and previous studies have revealed opposing results in the ability of NPs to induce or suppress oxidative stress, we subsequently wanted to determine if exposure the differently coated-MNPs could affect the oxidative stress resistance of $C$. elegans. In order to create an oxidative stress environment, C. elegans were treated for $24 \mathrm{hrs}$ with a low concentration of the chemical juglone, which has been shown to correlate with an increase in reactive oxygen species and oxidative damage [24].Worms were exposed to coated-MNPs or control 3-days prior to the juglone treatment and then placed back in the presence of coated-MNPs or control for the remaining 16 days of the assay. The control group had an average survival rate of $17 \%$, the carboxylMNPs $14 \%$, the amine-MNPs $24 \%$, and the polyaniline-MNPs $28 \%$. The trend in increased survival rate can be seen throughout the last 5 days of the assay, but is only significant on the last two days (Figure $3 \mathrm{~A}$ ). Surprisingly, the short $24 \mathrm{hr}$ low level exposure to oxidative stress resulted in divergent outcomes, as it appears that exposure to amineMNPs ( $\mathrm{p}=0.01)$ and polyaniline - MPNs $(\mathrm{p}=0.001)$ significantly aided C. elegans in resisting temporary oxidative stress, while those exposed to carboxyl-MNPs $(\mathrm{p}=0.08)$ had no effect when compared to the control group although the trend was similar to the other two MNPs up to day 19 (Figure 3B).

The increased ability of the C. elegans to resist a low level of oxidative stress exposure prompted us to further investigate if they would also respond in this positive manner to higher acute oxidative stress conditions when exposed to the coated-MNPs. For this assay
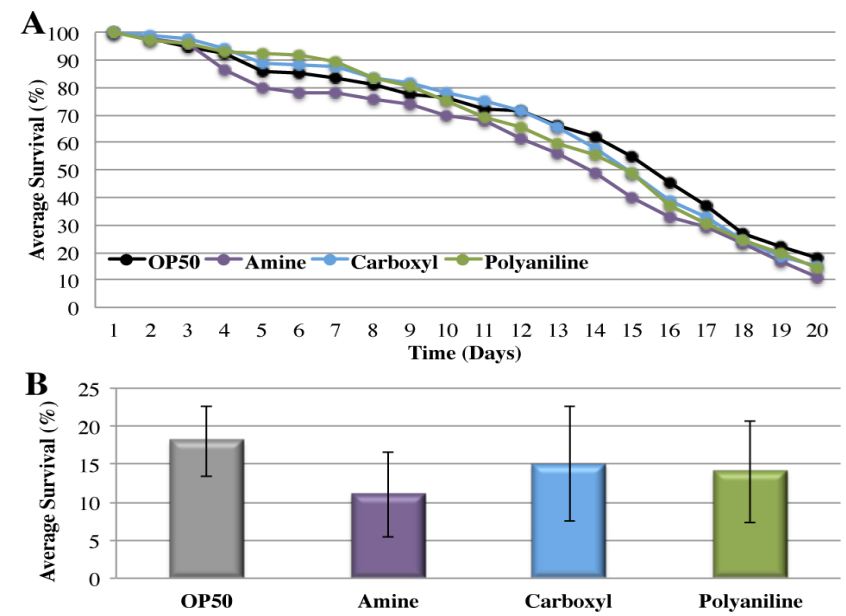

Figure 2: Coated-MNPs do not correlate with an impact on $C$. elegans longevity. (A) Longevity curves; (B) mean longevity. Each graph is the representative average of 3 different trials, $n=100$ worms per group. Error bars represent the standard deviation of the average. 
Citation: Callaway MK, Ochoa JM, Perez EE, Ulrich PE, Alocilja EC, and Vetrone SA, et al. (2013) Investigation of the Toxicity of Amine-coated, Carboxyl-coated and Polyaniline-coated FeO Magnetic Nanoparticles in Caenorhabditis elegans. J Biosens Bioelectron 4: 145. doi: $10.4172 / 2155-6210.1000145$

Page 4 of 5

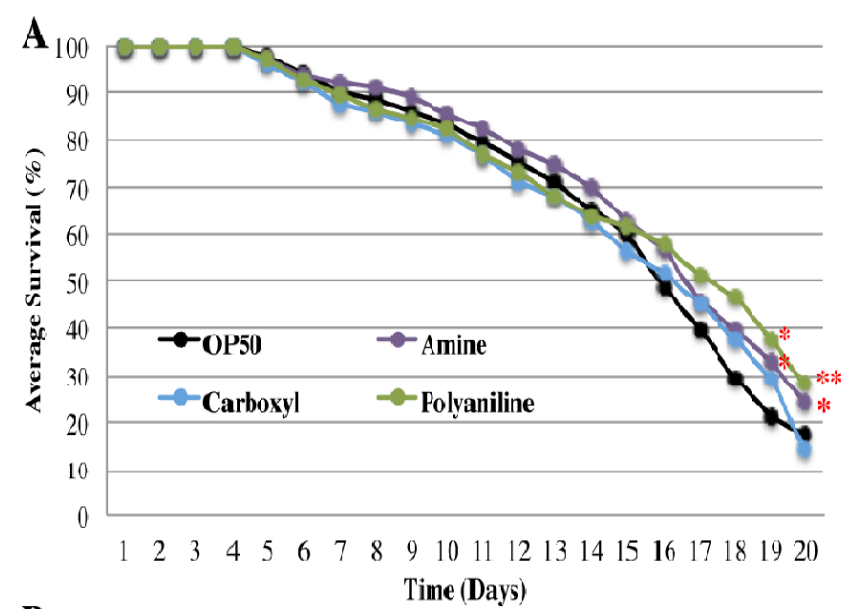

B

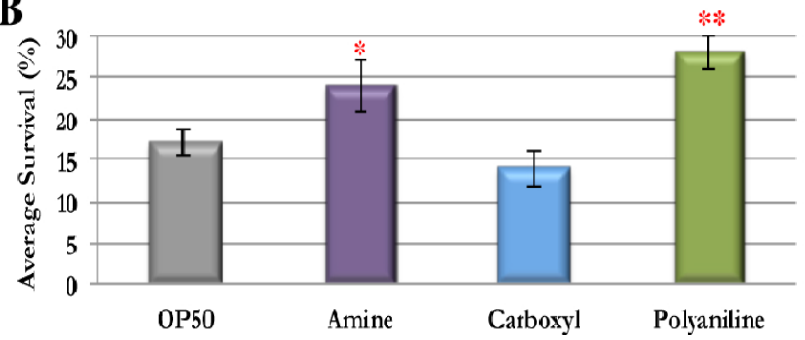

Figure 3: The ability of $C$. elegans to resistance temporary oxidative stress is statistically impacted by exposure to coated-MNP exposure. (A) Survival curves; (B) mean survival. Each graph is the representative average of 3 different trials, $n=100$ worms per group. Error bars represent the standard deviation of the average.

${ }^{*}$ represents $p \leq 0.01 ;{ }^{* *}$ represent $p \leq 0.001$, compared with control.

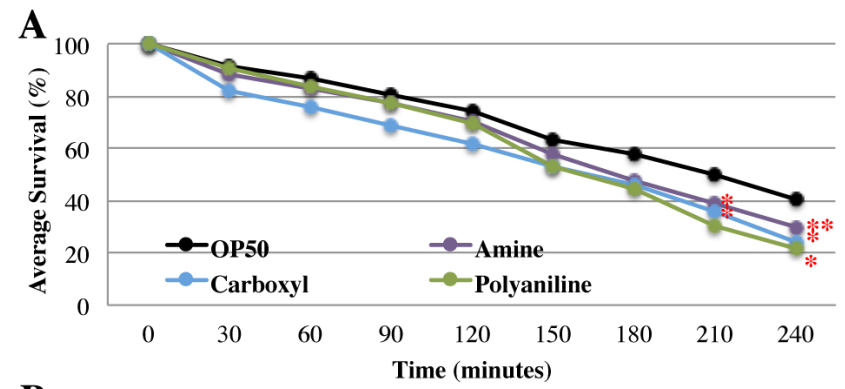

B

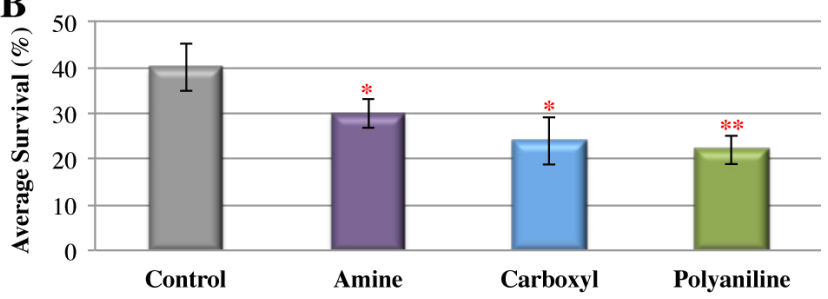

Figure 4: The ability of $C$. elegans to resistance acute oxidative stress is statistically decreased by exposure to coated-MNP. (A) Survival curves; (B) mean survival. Each graph is the representative average of 3 different trials, $n=100$ worms per group. Error bars represent the standard deviation of the average.

* represents $p \leq 0.01 ; \quad$ ** represent $p \leq 0.001$, compared with control.

we exposed the worms to coated-MNPs or control starting at the L4 stage until they were 2-day old adults, at which time they were treated to a high concentration of juglone to induce an acute oxidative stress environment. Interestingly, the findings of this assay demonstrated an inverse correlation with coated-MNP exposure and oxidative stress resistance (Figure 4). It can be clearly seen that throughout the $4 \mathrm{hr}$ exposure period the control group faired better at resisting the high oxidative stress environment which resulted in an overall significant improvement during the last two assessment points of the assay, as compared to the coated-MNP treated groups which showed a significantly decreased ability (Figure $4 \mathrm{~A}$ ). At the end of the assay on average the control group had a survival of $40 \%$ compared to the lower amine-MNPs $(30 \%)$, carboxyl-MNPs $(24 \%)$, and polyaniline-MNPs (22\%) groups (Figure 4B).

\section{Discussion}

To our knowledge, this is only the second study ever conducted that investigates the toxicity potential of coated-FeO -MNPs using the $C$. elegans nematode model. The findings of this study suggest that aminecoated, carboxyl-coated, and polyaniline-coated $\mathrm{FeO}$-MNPs can impart toxicity to the living C. elegans nematode when administered at a concentration of $100 \mu \mathrm{g} / \mathrm{mL}(100 \mathrm{mg} / \mathrm{L})$. In particular, the reduced defecation rate demonstrated in this study suggests that the carboxylMNPs and polyaniline-MPNs are having a harmful effect on the $C$. elegans. Interestingly, a 2012 study by Wu et al. investigating the toxicity of DMSA-coated FeO -MNPs on C. elegans also found an effect on defecation rate, but unlike our reduction in rate their study showed that the coated-MNPs resulted in an increase in defecation rate [17]. We hypothesize that the reduction achieved in our study might be occurring through the accumulation of coated-MNPs and eventual blockage within the intestinal cavity as has been previously shown by other studies investigating nanotoxicity of NPs [11,31].Moreover, our assessment of the impact on reproductive health fall in line with similar studies exploring the toxicity of NPs on reproduction health [32-34], as a recent study revealed that while the initial NP exposure was not harmful, the subsequent generations had decreased reproductive health that took as long as the fourth generation to show signs of recovery [35]

The assessment on longevity, although not significant, also displayed a trend towards reduced longevity that can be seen in the amineMNPs group, which was also unexpected, as these coated-MNPs had not shown any impact with our previous assays. We posit that the $7 \%$ difference in longevity between the amine-MNPs group and control did not result in a significant difference, as this difference can be attributed to the seemingly large deviation within worms of all groups, as similar studies have shown [14,31-33]. Lastly, our study also demonstrated that when C. elegans are exposed to coated-FeO -MNPs this correlated with a statistical reduction in their ability to resist oxidative stress. This outcome has also been shown in a similar study investigating the nanotoxicity of ceria NPs in C. elegans, which also demonstrated a reduced oxidative stress response when challenged with an acute oxidative stress environment induced by juglone administration [36]. Therefore, the outcomes of our temporary oxidative stress assay were unexpected, as past studies have shown either no effect or a reduction in oxidative stress resistance [14,32].

Taken together, our results demonstrating a statistical reduction in defecation rate and acute oxidative stress resistance, along with downward trends in egg production and longevity, indicate that precautions should be taken during the disposal of these NPs. Additionally, future efforts should focus on determining the mechanism through which these coated-MNPs are leading to a reduction in defecation rate, as well as explore the effect that they might have on multigenerational reproductive health. Equally, it would be a great 
Citation: Callaway MK, Ochoa JM, Perez EE, Ulrich PE, Alocilja EC, and Vetrone SA, et al. (2013) Investigation of the Toxicity of Amine-coated, Carboxyl-coated and Polyaniline-coated FeO Magnetic Nanoparticles in Caenorhabditis elegans. J Biosens Bioelectron 4: 145. doi: $10.4172 / 2155-6210.1000145$

Page 5 of 5

benefit to explore the impact of multiple nanoparticle exposure, as it is most likely that in a waste environment, these soil nematodes would be in direct contact with more than just one NP type.

\section{Acknowledgement}

This study is based upon work supported by the U.S. Department of Homeland Security under Grant Award Number 2010-ST-061-FD0001 through a grant awarded by the National Center for Food Protection and Defense at the University of Minnesota. The views and conclusions contained in this document are those of the authors and should not be interpreted as necessarily representing the officia policies, either expressed orimplied, of the U.S. Department of Homeland Security or the National Center for Food Protection and Defense.

\section{References}

1. Prathna TC, Lazar MN, Chandrasekaran AM, Mukherjee R, Mukherjee A (2010) Biomimetic Synthesis of Nanoparticles: Science, Technology and Applicability. Biomimetics Learning from Nature, Amitava Mukherjee (Ed.).

2. Zhang D, Huarng MC, Alocilja EC (2010) A multiplex nanoparticle-based biobarcoded DNA sensor for the simultaneous detection of multiple pathogens. Biosens Bioelectron 26: 1736-1742.

3. Setterington EB, Alocilja EC (2011) Rapid electrochemical detection of polyaniline-labeled Escherichia coli O157:H7. Biosens and Bioelectron 26: 2208-2214.

4. Vetrone SA, Huarng MC, Alocilja EC (2012) Detection of non-PCR amplified $\mathrm{S}$. enteritidis genomic DNA from food matrices using a gold-nanoparticle DNA biosensor: a proof-of-concept study. Sensors (Basel) 12: 10487-10499.

5. Doria G, Conde J, Veigas B, Giestas L, Almeida C, et al. (2012) Noble meta nanoparticles for biosensing applications. Sensors (Basel) 12: 1657-1687.

6. Kim J, Takahashi M, Shimizu T, Shirasawa T, Kajita M, et al. (2008) Effects of a potent antioxidant, platinum nanoparticle, on the lifespan of Caenorhabditis elegans. Mech Ageing and Dev 129: 322-331.

7. Li Y, Yu S, Wu Q, Tang M, Pu Y, et al. (2012) Chronic Al2O3-nanoparticle exposure causes neurotoxic effects on locomotion behaviors by inducing severe ROS production and disruption of ROS defense mechanisms in nematode Caenorhabditis elegans. J Hazard Mater 219-220: 221-30.

8. Wu Q, Nouara A, Li Y, Zhang M, Wang W, et al. (2013) Comparison of toxicities from three metal oxide nanoparticles at environmental relevant concentrations in nematode Caenorhabditis elegans. Chemosphere 90: 1123-1131.

9. Hunt PR, Marquis BJ, Tyner KM, Conklin S, Olejnik N, et al. (2013) Nanosilver suppresses growth and induces oxidative damage to DNA in Caenorhabditis elegans. J Appl Toxicol 33: 1131-1142.

10. Kovacic P, Somanathan R (2013) Nanoparticles: toxicity, radicals, electron transfer, and antioxidants. Methods Mol Biol 1028: 15-35.

11. Wang H, Wich R.L, Xing B (2009) Toxicity of nanoparticulate and bulk $\mathrm{ZnO}$ $\mathrm{Al} 2 \mathrm{O} 3$ and $\mathrm{TiO} 2$ to the nematode Caenorhabditis elegans. Environ Poll 157: 1171-1177.

12. Roh JY, Sim SJ, Yi J, Park K, Chung KH et al. (2009) Ecotoxicity of silver nanoparticles on the soil nematode Caenorhabditis elegans using functional ecotoxicogenomics. Environ Sci Technol 43: 3933-3940.

13. Tsyusko OV, Unrine JM, Spurgeon D, Blalock E, Starnes D, et al. (2012) Toxicogenomic responses of the model organism Caenorhabditis elegans to gold nanoparticles. Environ Sci Technol 46: 4115-4124.

14. Pluskota A, Horzowski E, Bossinger O, Mikecz A (2009) In Caenorhabditis elegans nanoparticle-bio-interactions become transparent: silica-nanoparticles induce reproductive senescence. PLoS 4: e6622.

15. Kolhatkar AG, Jamison AC, Litvinov D, Willson RC, Lee TR (2013) Tuning the magnetic properties of nanoparticles. Int J Mol Sci 14: 15977-16009.

16. Gomaa IO, Kader MH, Salah TA, Heikal OA (2013) Evaluation of in vitro mutagenicity and genotoxicity of magnetite nanoparticles. Drug Discov Ther 7: 116-123.

17. Wu Q, Li Y, Tang M, Wang D (2012) Evaluation of environmental safety concentrations of DMSA Coated Fe2O3-NPs using different assay systems in nematode Caenorhabditis elegans. PLoS One 7: e43729.

18. Yuk JS, Rose J, Alocilja EC (2010) Characterization of polyaniline-coated magnetic nanoparticles for application in a disposable membrane strip biosensor. EurPhys J Appl Phys 50: 11404 DOI: 10.1051/eohao/2010023
19. Setterington EB, Alocilja EC (2012) Electrochemical biosensor for rapid and sensitive detection of magnetically extracted bacterial pathogens. Biosensors 2: 15-31.

20. Nass R, Hamza I (2007) The nematode C. elegans as an animal model to explore toxicology in vivo: solid and axenic growth culture condition and compound exposure parameters. Curr Protoc Toxicol Chapter 1. Unit 1.9.

21. Leung MCK, Williams PL, Benedetto A, Au C, Helmchke KJ, et al. (2008) Caenorhabditis elegans: An emerging model in biomedical and environmental toxicology. Toxicological Sciences 106: 5-28.

22. Brenner S (1974) The genetics of Caenorhabditis elegans. Genetics 77: 71-94

23. Walker DS, Gower NJ, Ly S, Bradley GL, Baylis HA (2002) Regulated disruption of inositol 1, 4,5-triphosphate signaling in Caenorhabditis elegans reveals new functions in feeding and embryogenesis. Mol Biol Cell 13: 1329-1337.

24. Heidler T, Hartwig K, Daniel H, Wenzel U (2010) Caenorhabditis elegans lifespan extension caused by treatment with orally active ROS-generator is dependent on DAF-16 and SIR-2.1. Biogerontology 11: 183-195.

25. Branicky R, Hekimi S (2006) What keeps C. elegans regular: the genetics of defecation. Trends Genet 22: 571-579.

26. Bolanowski MA, Russell RL, Jacobson LA (1981) Quantitative measures of aging in the nematode Caenorhabditis elegans. I. Population and longitudinal studies of two behavioral parameters. Mech Ageing Dev 15: 279-295

27. Wu Q, Qu Y, Li X, Wang D (2012) Chromium exhibits adverse effects at environmental relevant concentrations in chronic toxicity assay system of nematode Caenorhabditis elegans. Chemosphere 87: 1281-1287.

28. Ma H, Bertsch PM, Glenn TC, Kabengi NJ, Williams PL (2009) Toxicity of manufactured zinc oxide nanoparticles in the nematode Caenorhabditis elegans. Environ Toxicol Chem 28: 1324-1330.

29. Sun J, Zhang Q, Wang Z, Yan B (2013) Effects of nanotoxicity on female reproductivity and fetal development in animal models. Int J Mol Sci 14: 9319 9337

30. Riddle DL, Blumenthal T, Meyer BJ, Priess JR (1997) C. elegans II, 2nd ed. Cold Spring Harbor Laboratory Press: New York.

31. Zhao Y, Wu Q, Tang M, Wang D (2013) The in vivo underlying mechanism for recovery response formation in nano-titanium dioxide exposed Caenorhabditis elegans after transfer to the normal condition. Nanomedicine.

32. Mohan N, Chen CS, Hsieh HH, Wu YC, Chang HC (2010) In vivo imaging and toxicity assessment of fluorescent nanodiamonds in Caenorhabditis elegans. Nano Lett 10: 3692-3699.

33. Zhang W, Sun B, Zhang L, Zhao B, Nie G, et al. (2011) Biosafety assessment of Gd@C82(OH)22 nanoparticles on Caenorhabditis elegans. Nanoscale 3: 2636-2641.

34. Zhou JC, Yang ZL, Dong W, Tang RJ, Sun LD, et al. (2011) Bioimaging and toxicity assessments of near-infrared upconversion luminescent NaYF4:Yb,Tm nanocrystals. Biomaterials 32: 9059-9067.

35. Kim SW, Kwak JI, An YJ (2013) Multigenerational study of gold nanoparticles in Caenorhabditis elegans: transgenerational effect of maternal exposure. Environ Sci Technol 47: 5393-5399.

36. Zhang H, He X, Zhang Z, Zhang P, Li Y, et al. (2011) Nano-CeO2 exhibits adverse effects at environmental relevant concentrations. Environ Sci Technol 45: $3725-3730$. 